\title{
PRECISE CALCULATION OF CROSS SECTIONS AND VOLUME FOR TREE STEM USING POINT CLOUDS
}

\author{
Shinsuke Eto ${ }^{1}$, Hiroshi Masuda ${ }^{1 *}$, Yuichiro Hiraoka ${ }^{2}$, Michinari Matsushita $^{2}$, Makoto Takahashi $^{2}$ \\ ${ }^{1}$ University of Electro-Communications \\ ${ }^{2}$ Forest Tree Breeding Center, Forestry and Forest Products Research Institute \\ e1932016@edu.cc.uec.ac.jp, h.masuda@uec.ac.jp \\ Commission II, WG II/3
}

KEY WORDS : Forest, Traits, terrestrial LiDAR, Point-Cloud, Stem Cross-Section, Stem Volume

\begin{abstract}
:
Woody biomass is an important parameter in forestry and forest research. In order to estimate of woody biomass, it is important to precisely and efficiently calculate section areas and volumes of tree stems in the forest. In this paper, we propose a method for calculating the cross-sectional area and the stem volume of trees from point clouds captured using the terrestrial laser scanner. In our method, each point cloud is converted into a wireframe model, and cross-section points are calculated as intersection between the wireframe and the horizontal planes placed at equal intervals. Cross-sectional shapes on each horizontal plane are approximated as nsided polygons and refined using the subdivision scheme. The section areas and stem volumes are calculated using the subdivision curves of stem contours. In our evaluation, our method could calculate section areas and stem volumes of trees with sufficient accuracy in practical use.
\end{abstract}

\section{INTRODUCTION}

Woody biomass is an important parameter in forestry and forest research (Hanaoka, et al., 2005, Lauri, et al., 2014). For estimation of woody biomass, it is important to precisely and efficiently calculate section areas and volumes of tree stems in the forest. Recently, instead of manual measurement, a terrestrial laser scanner (TLS) is often used to automatically extract tree parameters. By using TLS, point clouds of trees can be captured densely and accurately.

In conventional methods, section areas and volumes are typically calculated as approximated circles and cylinders. However, in actual trees, cross sections of trees may not be circles and the stems are not necessarily vertical, as shown in Figure 1 and 2. Therefore, in order to accurately calculate woody biomass, it is necessary to generate faithful models of $2 \mathrm{D}$ cross sections and 3D shapes of tree stems.

Researchers have developed methods for modeling cross section of trees. A traditional method is approximation using circles or ellipses (Simonse, et al., 2003, Aschoff, et al., 2004, Saito, et al., 2017). Other methods approximated cross sections using curves with a high degree of freedom, such as Fourier series curves (Di Wang, et al., 2017) and B-spline curves (Pfeifer, et al., 2004). However, such curves require a lot of calculation time for calculating many sections of each stem, and also require careful parameter settings.
Other researchers have also estimated woody biomass based on breast height diameters and tree species (Drexhage, et al., 2001, Yu, et al., 2013). However, in order to utilize their methods, it is necessary to investigate the relationship between breast height diameters and biomass for each tree species in a specific area.

In this paper, we propose a simple and robust method for representing cross sections of tree stems. Our method can efficiently obtain densely sliced cross sections of a large number of trees in the forest. The volume of a stem can be calculated using cross sections of each tree stem. For evaluating our method, we cut down trees and manually measured cross sections of tree stems after capturing point clouds of the trees using the terrestrial laser scanner. Then, the cross-sectional areas calculated using point clouds are compared to the areas obtained by manual measurement.
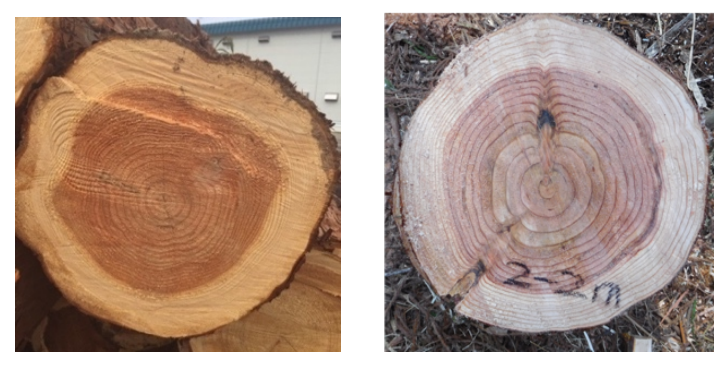

Figure 1. Cross section of tree

* Corresponding author 


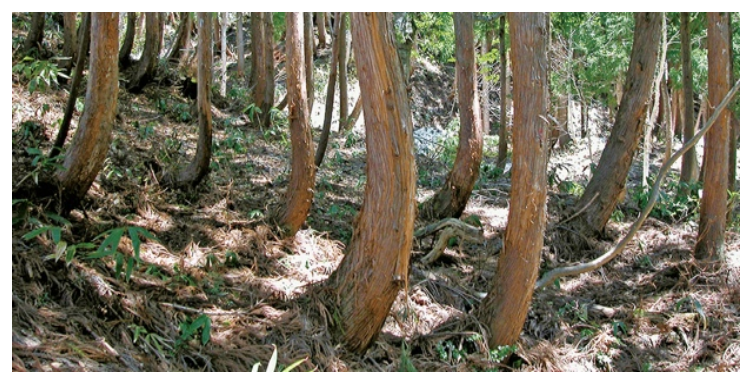

Figure 2. Non-vertical trees

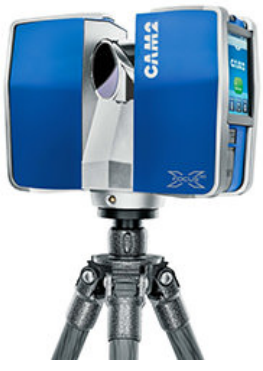

Figure 3. FARO Focus 3D X330

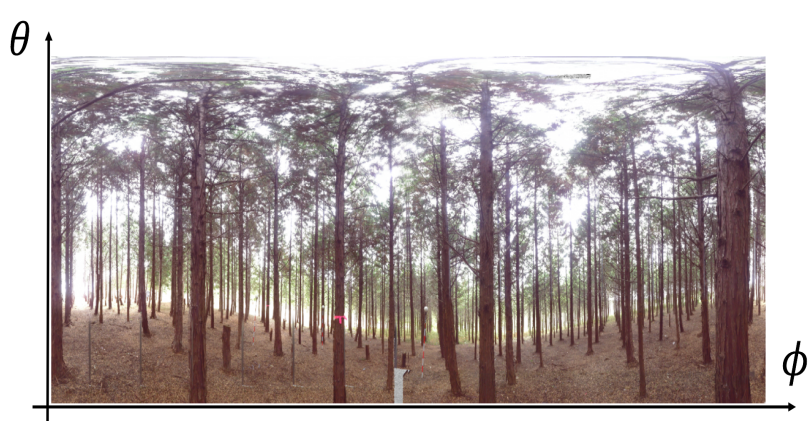

Figure 4. 2D Mapping of a point cloud

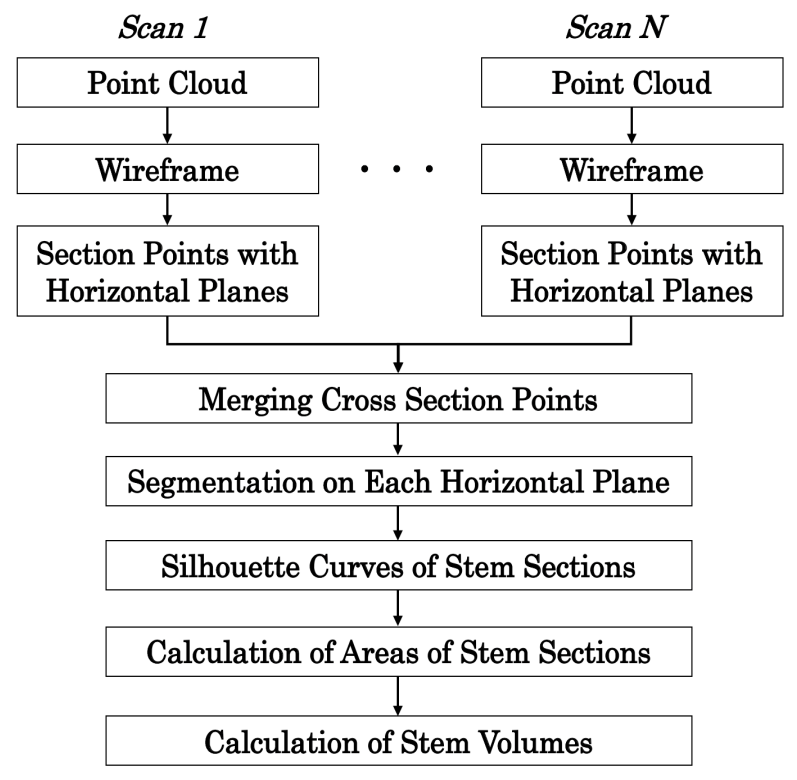

Figure 5. Calculation Process

\section{OVERVIEW}

The target tree species in this study is Japanese cedar, which is an evergreen conifer native to Japan, and is used as timber. The Japanese cedar forest is located at the Forest Tree Breeding Center in Hitachi City, Ibaraki Prefecture.

The terrestrial laser scanner used in this research was FARO Focus 3D X330, as shown in Figure 3. The laser of this laser scanner is class 1 and the wavelength is $1550 \mathrm{~nm}$. In this study, point clouds were captured with the angle resolution of 0.018 degree.

Since the laser beams rotate vertically and horizontally at the equal angle interval, points are ordered in the elevation angle $\phi$ and the azimuth angle $\theta$. Therefore, each point cloud can be mapped on the 2D grid, as shown in Figure 4. By connecting adjacent points on the $2 \mathrm{D}$ grid, a point cloud can be converted into a wireframe model.

Figure 5 shows the process for calculating cross sections and stem volumes. In this study, the Japanese cedar forest was measured at multiple positions. The total number of points was about 1.2 billion.

Each point cloud is converted into a wireframe model by connecting adjacent points on the $2 \mathrm{D}$ grid as line segments. Then, the wireframe is sliced using horizontal planes placed at equal intervals. In our method, intervals can be arbitrary determined regardless of point density, because trees are represented as a set of line segments. Cross-section points on each horizontal plane can be obtained by calculating intersections between line segments and the horizontal plane.

Next, cross-section points calculated from all point clouds are unified on each horizontal plane, and they are segmented into groups of neighbour points. Points in each group are regarded as a candidate stem section. Then, each group is approximated as a n-sided polygon, and the polygon is refined using the subdivision scheme. The subdivision scheme is a very robust and efficient way to obtain a smooth curve that interpolates vertices of a polygon.

The areas and volumes are calculated using subdivision curves of stem sections. The stem volume can be calculated using the section areas and the section intervals.

\section{METHOD}

\subsection{Cross section points}

Since point density varies depending on measurement conditions and the distances from the laser scanner, we convert each point cloud into a wireframe model to calculate section shapes at arbitrary intervals. 


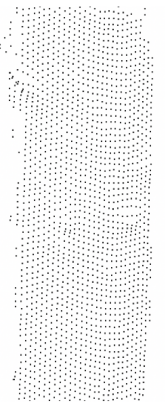

(a) Points

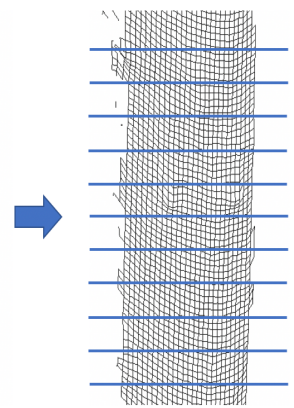

(b) Wireframe

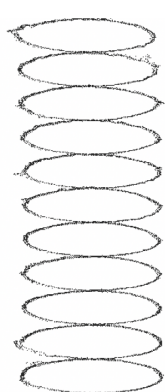

(c) Section points
Figure 6. Cross section points with horizontal planes

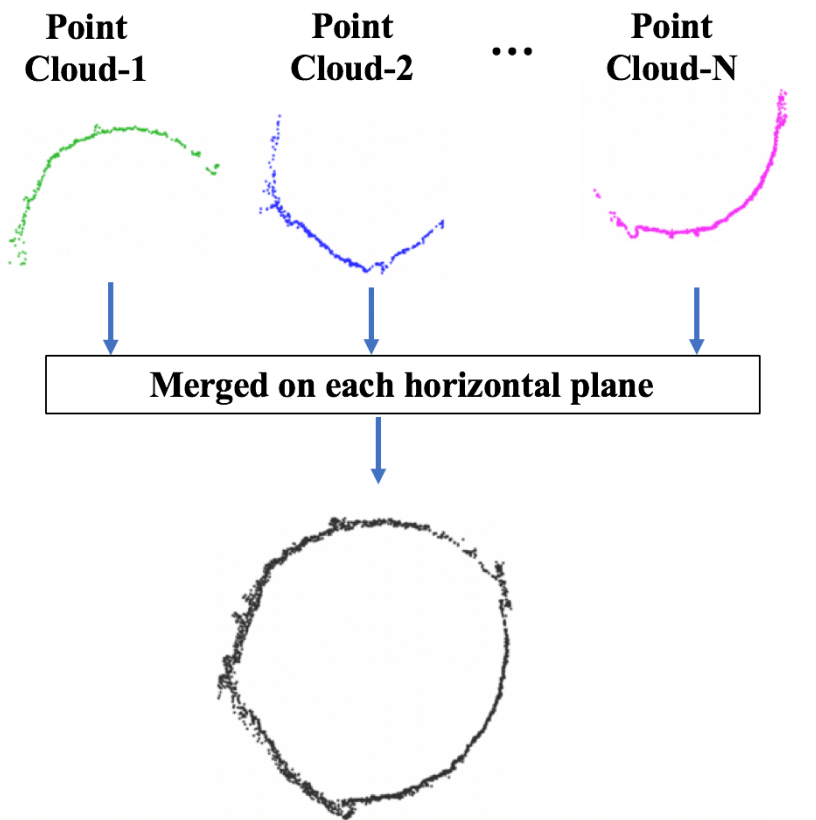

Figure 7. Merging cross-sectional points

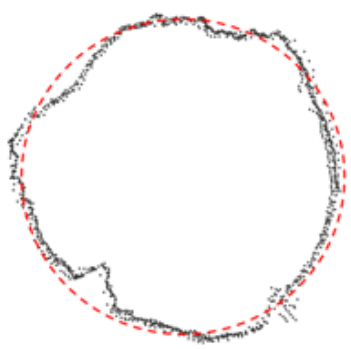

(a) Circle fitting

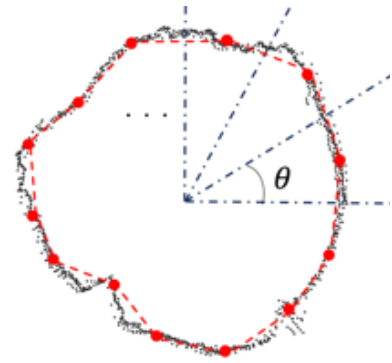

(b) Point sampling
Figure 8. Sampling points on section shape

A point cloud captured using the terrestrial laser scanner can be mapped on the 2D grid, as shown in Figure 4. Therefore, line segments can be generated by connecting adjacent points on the 2D grid if the distances are less than thresholds. Since the 2D grid is generated for each point cloud, multiple wireframe models are generated for point clouds captured at multiple scanner positions.

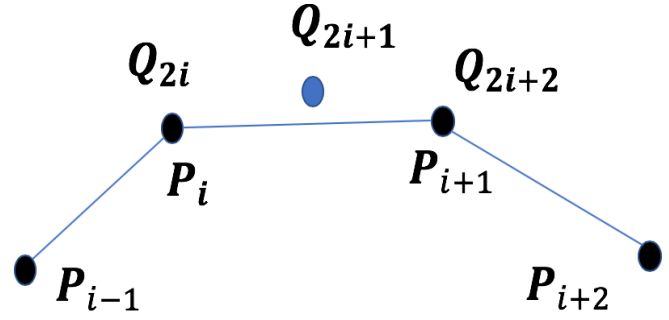

Figure 9. Four-point subdivision scheme

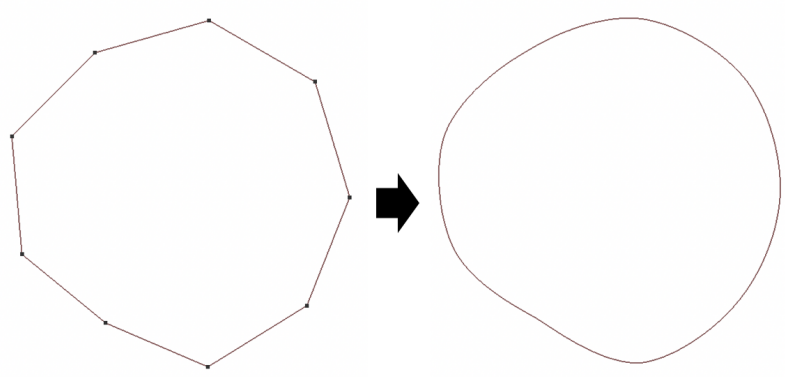

(a) 9-sided polygon

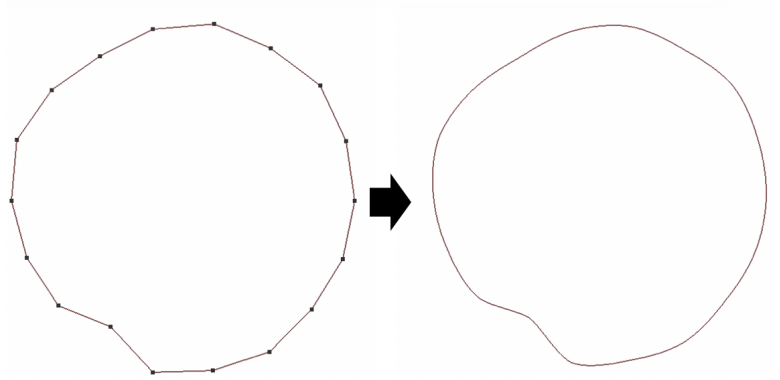

(b) 18-sided polygon

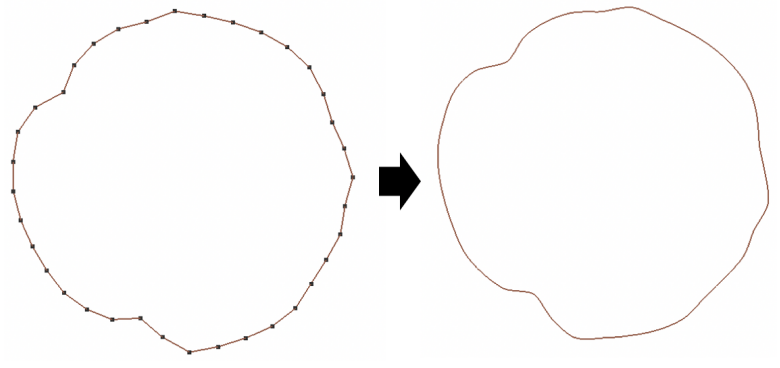

(b) 36-sided polygon

Figure 10. Application of four-point subdivision scheme

Then, horizontal planes are placed at equal intervals, cross section points are calculated between a wireframe and the horizontal planes, as shown in Figure 6. Since a wireframe consists of line segments, cross section points can be efficiently calculated between line segments and horizontal planes.

Cross section points are merged on each horizontal plane, as shown in Figure 7. Since a large number of stem sections exist on each horizontal plane, section points are segmented by using the neighbor search. When the distance between two points is less than a threshold, these points are segmented into the same group. In this study, the threshold was specified as $5 \mathrm{~cm}$. 


\subsection{Representation of section shape}

While most conventional methods approximated each section as a circle or an ellipse, as shown in Figure 8 (a), our method approximates the section as a n-sided polygon by sampling $n$ points from merged cross section points. Since section points are noisy, points are sampled as the median points in fans with angle $\theta$, as shown in Figure 8 (b). In this study, the angle was specified as 10 degree. By using the sampled points, the section shape is represented as a n-sided polygon.

\subsection{Refining by subdivision scheme}

Since the area of a n-sided polygon is a little smaller than the actual section area, we increase the number of points using the four-point subdivision scheme (Dyn, 1992). Figure 9 shows the four-point subdivision scheme, which doubles the number of vertices of a polygon. In this figure, $\left\{\mathbf{Q}_{j}\right\} \quad(j=1,2, \ldots, 2 n)$ are calculated from $\left\{\mathbf{P}_{i}\right\}(i=1,2, \ldots, n)$ using the following equations.

$$
\left\{\begin{array}{l}
\boldsymbol{Q}_{2 i}=\boldsymbol{P}_{i} \\
\boldsymbol{Q}_{2 i+1}=\frac{-1}{16} \boldsymbol{P}_{i-1}+\frac{9}{16} \boldsymbol{P}_{i}+\frac{9}{16} \boldsymbol{P}_{i+1}+\frac{-1}{16} \boldsymbol{P}_{i+2}
\end{array}\right.
$$

By applying this subdivision scheme many times, a polygon converges on a smooth spline curve. Figure 10 shows section curves generated using the four-point subdivision scheme. In this figure, 9-sided, 18-sided, and 36 sided polygons are converted into smooth subdivision curves.

\subsection{Calculation of stem volume}

Volumes can be calculated from section areas. Suppose the cross sections are calculated using $n$ horizontal planes placed at the interval $h$, and the calculated section areas are denoted as $\left\{S_{i}\right\}(i=1,2, \ldots, n)$. Then the stem volume $V$ can be calculated using the following equation.

$$
V=\sum_{i=1}^{n-1} \frac{h}{3}\left(S_{i}+S_{i+1}+\sqrt{S_{i} S_{i+1}}\right)
$$

The accuracy of the volume increases as the number of sections increases. In this study, stem sections are calculated at the interval of $5 \mathrm{~cm}$.

\section{EVALUATION}

\subsection{Evaluation of smoothed section curves}

First, the areas of subdivision curves are compared to the ones of n-sided polygons. In this evaluation, two stem sections were selected, and they are represented as n-sided polygons and subdivision curves. Then, their areas were calculated. Figure 11 shows the selected section shapes.

Table 1 shows the areas of stem sections approximated as 9sided, 18-sided, and 36-sided polygons. These polygons were converted to subdivision curves using the four-point subdivision scheme, and their areas were calculated. The values in

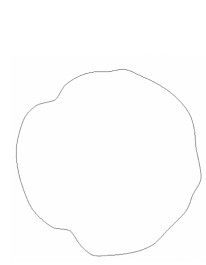

(a) Section A

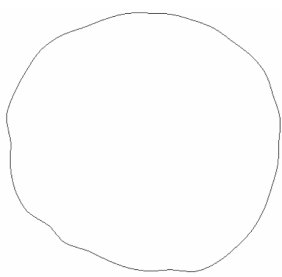

(b) Section B
Figure 11. Section shapes

\begin{tabular}{|c|c|c|c|c|}
\hline \multirow{2}{*}{ Section } & Shape & \multicolumn{3}{|c|}{ Area $\left(\mathrm{cm}^{2}\right)$} \\
\cline { 3 - 5 } & Type & $\mathrm{n}=9$ & $\mathrm{n}=18$ & $\mathrm{n}=36$ \\
\hline \multirow{4}{*}{$\mathrm{A}$} & n-sided & 230.4 & 264.4 & 275.9 \\
& polygon & $(-19.3 \%)$ & $(-7.4 \%)$ & $(-3.4 \%)$ \\
\cline { 2 - 5 } & Subdivision & 280.0 & 285.6 & 285.6 \\
& curve & $(-1.9 \%)$ & $(0.0 \%)$ & $(0.0 \%)$ \\
\hline \multirow{4}{*}{ B } & n-sided & 739.2 & 838.9 & 881.9 \\
& polygon & $(-18.8 \%)$ & $(-7.9 \%)$ & $(-3.2 \%)$ \\
\cline { 2 - 5 } & Subdivision & 899.6 & 905.0 & 910.9 \\
& curve & $(-1.2 \%)$ & $(-0.6 \%)$ & $(0.0 \%)$ \\
\hline
\end{tabular}

Table 1. Areas of section shapes

parentheses indicate the differences from the actual areas.

The areas of polygons were smaller than the actual area, and the accuracy increased as the number of sides of polygons increased. By refining polygons to subdivision curves, the accuracy of areas has improved in all cases. In this evaluation, polygons with 18 or more sides were sufficiently accurate by refining the subdivision scheme.

This evaluation shows that it is reasonable to maintain stem sections as polygons and refine the polygons when calculating traits of trees.

\subsection{Comparison of section areas with manual measurement}

We compared the calculated section areas with manually measured section areas. We selected five trees from the forest in the Forest Tree Breeding Center (Figure 12). The point clouds of the selected trees are shown in Figure 13. After capturing point clouds in the forest, we cut down the selected trees and sliced each tree stem every $1 \mathrm{~m}$. Then, we copied the contours of section shapes on tracing papers, and measured the areas of actual section shapes as precisely as possible.

In this evaluation, section shapes of each stem were calculated at the interval of $5 \mathrm{~cm}$. Figure 14 shows section shapes of a tree stem. Each section shape was approximated as a 36-sided polygon and refined into a subdivision curve by applying the four-point subdivision scheme four times.

Table 2 shows the section areas at the breast height for the selected tree stems. This result shows that our method could calculate section areas with sufficient accuracy for practical use. In most cases, the calculated areas were larger than manually measured areas. This is because the moss and leaves around stems were regarded as points of stem surfaces. 


\subsection{Evaluation of stem volumes}

We calculated stem volumes and compared to manual measurement. Stem volumes were calculated using Equation 2. Table 3 shows the volumes of the selected trees. In most cases, the accuracy of volumes could be calculated with sufficient accuracy.

The differences of volumes were relatively large for stems No. 3 and No. 5. Since the sea breeze was blowing on the day of measurement, the upper parts of trees with small diameters swayed in the wind. Since the diameters of stems No. 3 and No.

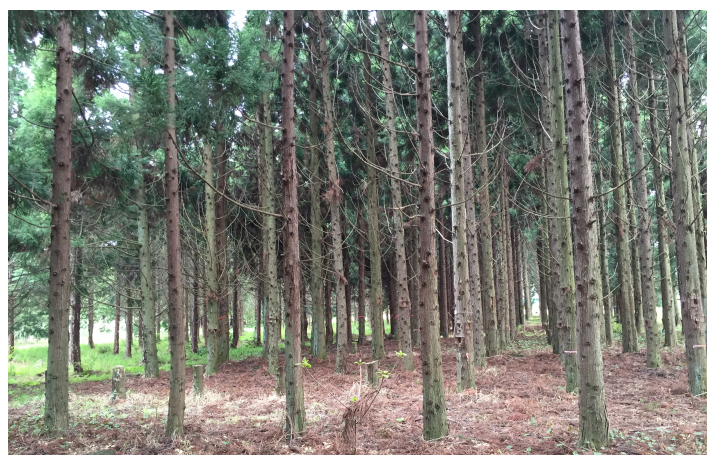

Figure 12. Forest in the Forest Tree Breeding Center

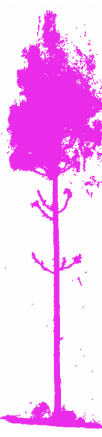

(No.1)

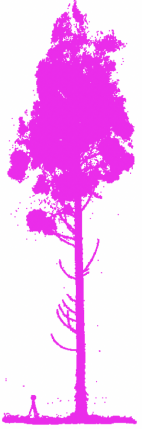

(No. 2)

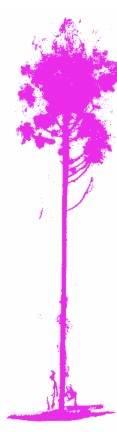

(No. 3)

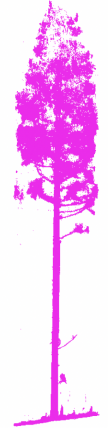

(No.4)

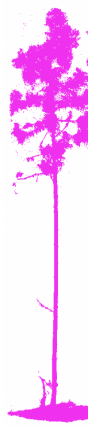

(No. 5)
Figure 13. Point clouds of selected trees
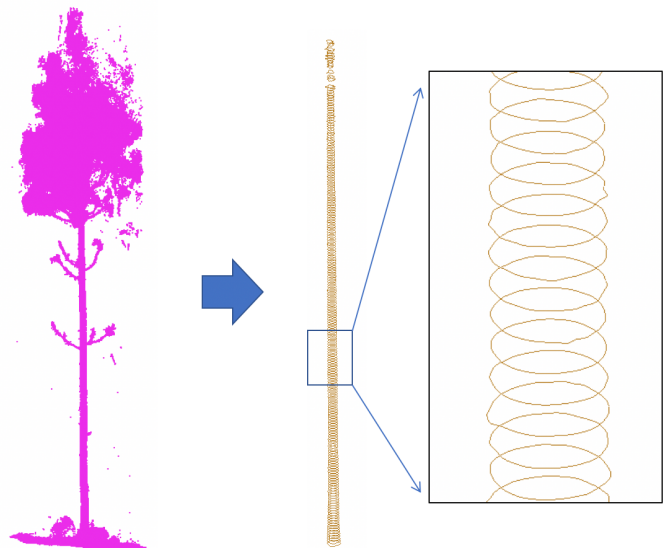

Figure 14. Sections calculated from point cloud
5 were relatively small, it is considered that the stems largely swayed during laser scanning.

Table 4 shows the volume of tree No. 5 in the height range of 1 $\mathrm{m}$. The difference from manual measurement increases as the height increases and the diameter decreases. When a tree sways in the wind, the point cloud is distributed over a wider range than it actually is, and therefore, the section area and the stem volume become larger than the actual area.

This evaluation revealed the problem of measuring trees using the terrestrial laser scanner. The limitation of our method is that it is difficult to calculate the correct areas and volumes of trees when trees largely sway due to the wind.

\begin{tabular}{|c|c|c|c|}
\hline \multirow{2}{*}{ Tree } & \multicolumn{2}{|c|}{ Section Areas $\left(\mathrm{cm}^{2}\right)$} & \multirow{2}{*}{ Difference } \\
\cline { 2 - 3 } & $\begin{array}{c}\text { Calculated from } \\
\text { Point Clouds }\end{array}$ & $\begin{array}{c}\text { Manual } \\
\text { Measurement }\end{array}$ & \\
\hline No. 1 & 280.3 & 285.1 & $-1.7 \%$ \\
\hline No. 2 & 930.5 & 909.4 & $+2.3 \%$ \\
\hline No. 3 & 209.1 & 204.6 & $+2.2 \%$ \\
\hline No. 4 & 359.6 & 353.6 & $+1.7 \%$ \\
\hline No. 5 & 219.5 & 226.7 & $+3.3 \%$ \\
\hline
\end{tabular}

Table 2. Section areas at the breast height

\begin{tabular}{|c|c|c|c|}
\hline \multirow[b]{2}{*}{ Tree } & \multicolumn{2}{|c|}{ Section Volumes $\left(m^{3}\right)$} & \multirow[b]{2}{*}{ Difference } \\
\hline & $\begin{array}{c}\text { Calculated from } \\
\text { Point Clouds }\end{array}$ & $\begin{array}{c}\text { Manual } \\
\text { Measurement }\end{array}$ & \\
\hline No. 1 & 0.0169 & 0.0165 & $+2.4 \%$ \\
\hline No. 2 & 0.0681 & 0.0662 & $+2.7 \%$ \\
\hline No. 3 & 0.0155 & 0.0147 & $+5.7 \%$ \\
\hline No. 4 & 0.0232 & 0.0226 & $+2.5 \%$ \\
\hline No. 5 & 0.0145 & 0.0130 & $+10.1 \%$ \\
\hline
\end{tabular}

Table 3. Stem volumes of selected trees

\begin{tabular}{|c|c|c|c|}
\hline \multirow{2}{*}{$\begin{array}{c}\text { Height } \\
\text { Range }\end{array}$} & \multicolumn{2}{|c|}{ Section Volumes $\left(\mathrm{m}^{3}\right)$} & \multirow{2}{*}{\begin{tabular}{c} 
Difference \\
\cline { 2 - 3 }
\end{tabular}} \\
\hline 0 Point Clouds & $\begin{array}{c}\text { Manual } \\
\text { Measurement }\end{array}$ & \\
\hline $0-1 \mathrm{~m}$ & 0.00220 & 0.00213 & $+3.3 \%$ \\
\hline $1-2 \mathrm{~m}$ & 0.00206 & 0.00199 & $+3.3 \%$ \\
\hline $2-3 \mathrm{~m}$ & 0.00188 & 0.00179 & $+5.3 \%$ \\
\hline $3-4 \mathrm{~m}$ & 0.00178 & 0.00168 & $+5.5 \%$ \\
\hline $4-5 \mathrm{~m}$ & 0.00171 & 0.00160 & $+6.9 \%$ \\
\hline $5-6 \mathrm{~m}$ & 0.00165 & 0.00144 & $+14.0 \%$ \\
\hline $6-7 \mathrm{~m}$ & 0.00159 & 0.00126 & $+26.5 \%$ \\
\hline $7-8 \mathrm{~m}$ & 0.00160 & 0.00111 & $+44.3 \%$ \\
\hline
\end{tabular}

Table 4. Volume in each height range for tree No. 5 


\section{CONCLUSION}

In this paper, we proposed a method to represent section shapes of stems using subdivision curves. In our method, each point cloud was mapped on the $2 \mathrm{D}$ grid, and it was converted to a wireframe model by connecting adjacent points. Then, section points were calculated as intersections between the wireframe and horizontal planes. Section points calculated for each point cloud were merged on each horizontal plane, and the merged points were segmented to each stem section. Section shapes of a stem were represented as n-sided polygons, and the polygons were refined as subdivision curves using the four-point scheme. Then, section areas and stem volumes were calculated using the subdivision curves. In experiments, section areas and volumes could be calculated with sufficient accuracy for practical use. However, for trees with small diameters, the stem volume might have a $10 \%$ or more error when trees swayed due to the wind.

In future work, we would like to develop methods for estimating correct section areas of upper parts of trees. In addition, we would like to develop robust registration methods to increase the accuracy of section shapes.

\section{REFERENCES}

Aschoff, T., \& Spiecker, H. (2004). Algorithms for the automatic detection of trees in laser scanner data. International Archives of Photogrammetry, Remote Sensing and Spatial Information Sciences, 36(Part 8), W2.

Drexhage, M., \& Colin, F. (2001). Estimating root system biomass from breast-height diameters. Forestry, 74(5), 491-497.

Dyn, N. (1992). Subdivision schemes in CAGD. Advances in numerical analysis, 2, 36-104.

Hanaoka, T., Inoue, S., Uno, S., Ogi, T., \& Minowa, T. (2005). Effect of woody biomass components on air-steam gasification. Biomass and bioenergy, 28(1), 69-76.

Lauri, P., Havlík, P., Kindermann, G., Forsell, N., Böttcher, H., \& Obersteiner, M. (2014). Woody biomass energy potential in 2050. Energy Policy, 66, 19-31.

Pfeifer, N., \& Winterhalder, D. (2004). Modelling of tree cross sections from terrestrial laser scanning data with free-form curves. International Archives of Photogrammetry, remote sensing and spatial information sciences, 36(Part 8), W2.

Saito, K., Masuda, H., Hiraoka, Y., Matsushita, M., \& Takahashi, M. (2017). Shape reconstruction of trees in massive-scale forests for evaluation of morphological traits, SilviLaser 2017.

Simonse, M., Aschoff, T., Spiecker, H., \& Thies, M. (2003, September). Automatic determination of forest inventory parameters using terrestrial laser scanning. In Proceedings of the scandlaser scientific workshop on airborne laser scanning of forests (Vol. 2003, pp. 252-258). Umeå: Sveriges Lantbruksuniversitet.

Wang, D., Kankare, V., Puttonen, E., Hollaus, M., \& Pfeifer, N. (2017). Reconstructing stem cross section shapes from terrestrial laser scanning. IEEE Geoscience and Remote Sensing Letters, 14(2), 272-276.

Yu, X., Liang, X., Hyyppä, J., Kankare, V., Vastaranta, M., \& Holopainen, M. (2013). Stem biomass estimation based on stem reconstruction from terrestrial laser scanning point clouds. Remote Sensing Letters, 4(4), 344-353. 